\title{
Enhancer of Zeste homolog 2 (EZH2) is overexpressed in recurrent nasopharyngeal carcinoma and is regulated by miR-26a, miR-101, and miR-98
}

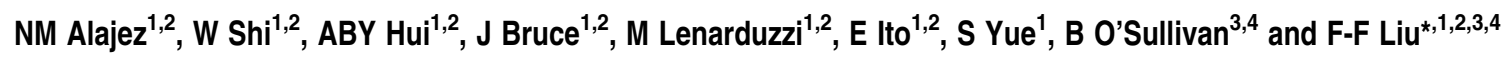

There is increasing evidence supporting the role of members of the polycomb group (PcG) gene family in tumor development and progression. However, their precise role in tumorigenesis and mechanisms of their regulation remain to be elucidated. Using nasopharyngeal carcinoma (NPC) as a disease model, a comprehensive analysis was undertaken on the clinical significance of EZH2 expression, identification of the cellular processes regulated by EZH2, and the mechanisms of its deregulated expression. Herein, we report EZH2 as being associated with a higher risk of relapse in NPC patients $(P=0.002)$. Genome-wide microarray and bioinformatics identified several vital cellular processes (such as differentiation, development, and apoptosis) to be regulated by EZH2, corroborated by in vitro lethality, and delayed tumor formation in vivo upon EZH2 depletion. The combination of global microRNA (miR) profiling in primary NPC specimens, and in silico analyses provided several candidate miRs that could regulate EZH2. Using a luciferase-based assay, miR-26a, miR-101, and miR-98 were validated as bona fide regulators of EZH2 expression. In particular, miR-98 was underexpressed in relapsed patient samples, strongly suggesting an important role for the miR-98 and EZH2 axis in NPC biology.

Cell Death and Disease (2010) 1, e85; doi:10.1038/cddis.2010.64; published online 21 October 2010

Subject Category: Cancer

There is increasing evidence supporting the role for members of the polycomb group (PcG) family in tumor development and progression. ${ }^{1-3}$ The PcG family of genes mediates their function by the formation of several multimeric polycomb repressive complexes (PRCs) PRC1, PRC2, PRC3, or PRC4. ${ }^{4}$ In embryonic and adult stem cells, PcG genes maintain cellular identity by repressing transcription of key developmental genes, such as homeobox genes, and preventing senescence through repression of the INK4A locus. ${ }^{5,6} \mathrm{EZH} 2$ histone methyl transferase, a member of the PcG family, is the catalytic subunit of the PRC2, which is responsible for methylating lysine-27 on histone-3. This epigenetic modification of $\mathrm{H} 3$ is necessary for gene repression through the PRC2 complex. ${ }^{7}$ In different systems, PRC2 and PRC1 appear to cooperatively mediate gene silencing. ${ }^{8}$ Although more is known about the function of PcG family during development, their roles in human malignancies continue to evolve.

MicroRNAs (miRs) are endogenous 19-25 nucleotide noncoding RNAs, which regulate gene expression in a sequencespecific manner. Since their initial identification in the early 90 s, miRs are now increasingly recognized to be key regulators of gene expression in several biological systems, including cancer. ${ }^{9}$ The current understanding is that miRs regulate gene expression by mediating mRNA target degradation and inhibition of translation (reviewed in Calin GA et al. ${ }^{10}$ )
Nasopharyngeal carcinoma (NPC) represents a subset of head and neck epithelial cancers, treated by radiation therapy for early disease, but combined with chemotherapy for more advanced stages. Despite progress in NPC management, a significant proportion of patients still develop distant metastases, underscoring the need for an improved understanding of the molecular mechanisms underlying therapy failure. We have recently reported that overexpression of BMI-1, another member of the PcG family, mediated radiation resistance through repression of $\mathrm{P} 53$ and reactive oxygen species (ROS)-dependent apoptosis. ${ }^{11}$ In this study, the clinical significance of EZH2 in NPC was also determined, along with its mechanisms in driving NPC progression, and finally, its own regulation, in the context of miRs.

\section{Results}

Overexpression of EZH2 is associated with poor clinical outcome in NPC. Our previous microarray expression profiling study on NPC demonstrated overexpression of $\mathrm{EZH} 2,{ }^{12}$ an observation which was followed up in the current study using immunohistochemistry. EZH2 protein expression was, hence, assayed in an independent collection of 39 archival NPC specimens (cohort 'A'), demonstrating tumor nuclear immunostaining (Figure 1a). Using a relative scoring

\footnotetext{
${ }^{1}$ Division of Applied Molecular Oncology, Ontario Cancer Institute, Toronto, Canada; ${ }^{2}$ Department of Medical Biophysics, University of Toronto, Toronto, Canada; ${ }^{3}$ Department of Radiation Oncology, University Health Network, Toronto, Canada and ${ }^{4}$ Department of Radiation Oncology, University of Toronto, Toronto, Canada ${ }^{*}$ Corresponding author: F-F Liu, Department of Radiation Oncology, Princess Margaret Hospital/Ontario Cancer Institute, 610 University Avenue, Toronto, Ontario, M5G 2M9 Canada. Tel: + 416946 2123; Fax: + 416946 4586; E-mail: Fei-Fei.Liu@ rmp.uhn.on.ca Keywords: EZH2; microRNA; nasopharyngeal carcinoma (NPC)

Abbreviations: PcG, polycomb group; EZH2, enhancer of Zeste homologue 2; NPC, nasopharyngeal carcinoma; miR, microRNA; Hox, homeobox; PRC, polycomb repressive complex; RT, radiation therapy; siRNA, small interference RNA; dsb, double strand break; ROS, reactive oxygen species; FFPE, formalin-fixed and paraffinembedded

Received 21.5.10; revised 17.8.10; accepted 16.9.10; Edited by G Melino
} 
a
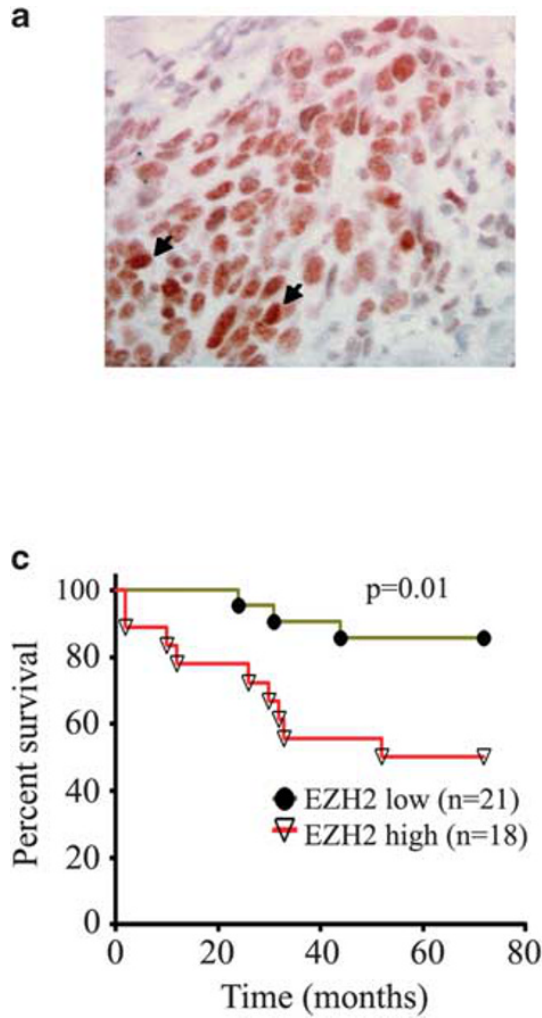
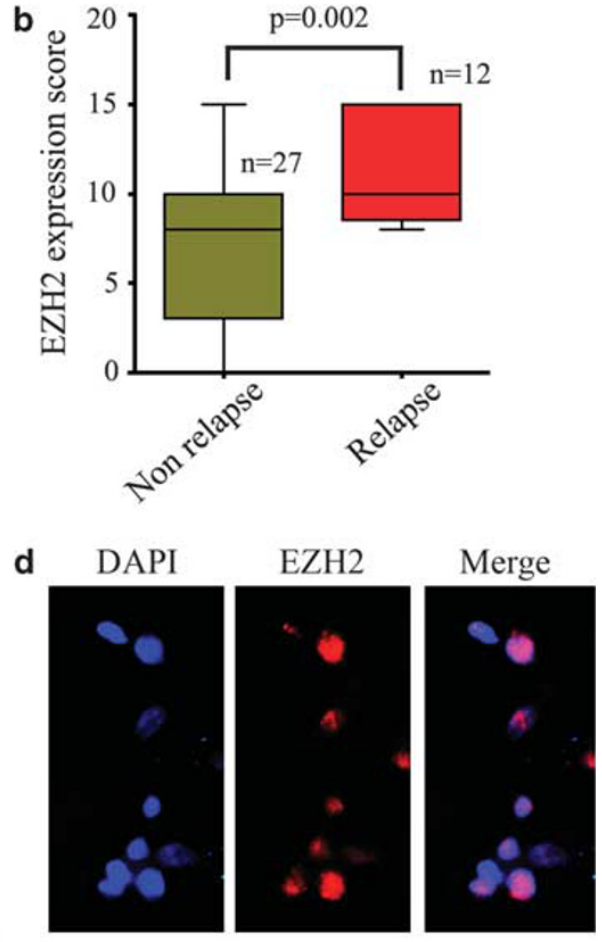

Figure 1 Overexpression of EZH2 is associated with poor clinical outcome in NPC (a) Representative immunohistochemical staining demonstrating expression of EZH2 (arrow) in the nuclei of NPC cells. (b) EZH2 is expressed at significantly higher levels in relapsed NPC compared with nonrelapsed patient tumors (cohort 'A'). (c) KaplanMeier plot of overall survival in the same cohort as a function of EZH2 expression, using the median score of $\geq 10$ (high) versus $<10$ (low). (d) Immunofluorescence overlay of EZH2 (red) and nuclear (blue) staining demonstrating nuclear expression of EZH2 in C666-1 cells

system of proportion and intensity of tumor nuclear staining, $\mathrm{EZH} 2$ overexpression was observed to associate with a higher probability of relapse (Figure $1 \mathrm{~b}, P=0.002$ ). This overexpression translated into reduced survival wherein the 5 -year actuarial probability of overall survival was $45 \%$ for high EZH2 expressors versus $82 \%$ for low expressors, using a median expression value of 10 as the cutoff (Figure 1c, $P=0.01)$. In the EBV-positive NPC cell line C666-1, which provides the NPC in vitro and in vivo model for the subsequent studies, strong nuclear expression of $\mathrm{EZH} 2$ was also observed (Figure 1d).

Targeted depletion of EZH2 is lethal to C666-1 NPC cells. Given its apparent role in NPC progression, EZH2 was evaluated for its function in NPC cell survival. To this end, EZH2 was knocked down using small interference RNA (siRNA), which resulted in reduced $\mathrm{EZH} 2$ expression at both the transcript and the protein levels (Figures $2 a$ and $b$ ), associated with decreased cell viability (Figure 2c). Cytotoxicity was further increased when cells were exposed to ionizing radiation (IR) (Figure $2 \mathrm{c}$, right panel). To confirm that the cytotoxicity was specifically attributable to EZH2 depletion, and not to off-target effects, these experiments were repeated using a previously described siRNA duplex targeting $\mathrm{EZH} 2{ }^{2}$, demonstrating a similar reduction in cell viability (Supplementary Figure 1A). A similar level of cytotoxicity was observed when EED was also depleted;
EED being a second component of the PRC2 complex, corroborating the importance of $\mathrm{PcG}$ genes in maintaining survival of NPC cells (Supplementary Figure 1B). Using combination index analysis, ${ }^{13}$ a more than additive interaction was observed between $\mathrm{EZH} 2$ depletion and IR (Figure 2d). Then we sought to determine if forced expression of EZH2 could protect normal cells from IR-induced cell death. Overexpression of EZH2 did not confer radiation resistance in normal oral epithelial (data not shown) cells, hence suggesting that the role of EZH2 in radiation sensitivity is context dependent and is mainly restricted to tumor cells.

EZH2 depletion induces apoptosis in C666-1 cells. Cell cycle analysis demonstrated that EZH2-depletion in C666-1 cells led to a significant increase in apoptosis (sub-G0), an increase in $\mathrm{G} 1$, and a decrease in the S-G2M phases of the cell cycle (Figure 3a). Concordantly, apoptosis was associated with caspase $3 / 7$ activation observed as early as $24 \mathrm{~h}$ post-transfection (Figure 3b). Given the observed interaction between $\mathrm{EZH} 2$ depletion and IR, the kinetics of DNA double-strand break (dsb) formation was assessed, using $\gamma-\mathrm{H} 2 \mathrm{AX}$ staining, a commonly utilized marker for dsb. ${ }^{14}$ Indeed, an increase in dsb was observed with EZH2 depletion alone, which was further increased on IR exposure (Figure $3 \mathrm{c}$ ), although the rate of $\gamma-\mathrm{H} 2 \mathrm{AX}$ reduction following IR was not affected by $\mathrm{EZH} 2$ depletion, indicating that $\mathrm{EZH} 2$ does not have an apparent role in dsb repair. 
a

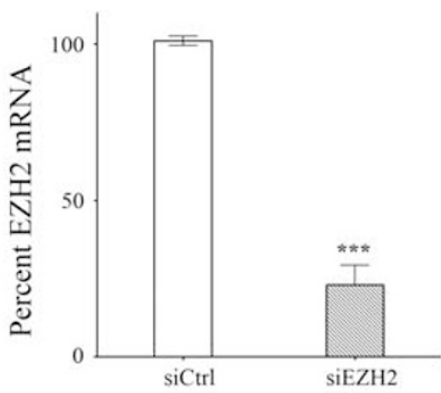

C

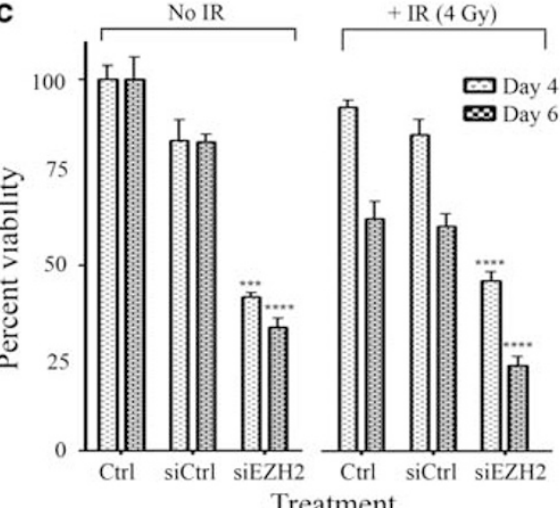

b

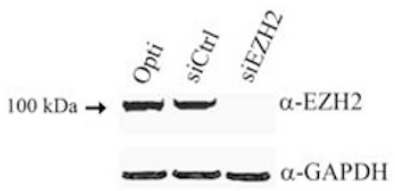

d

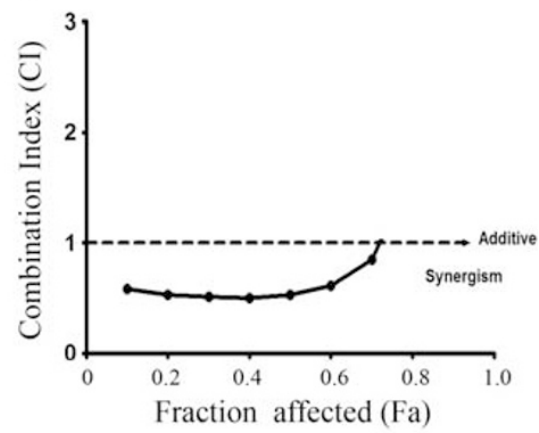

Figure 2 EZH2 is important for survival of NPC cells in vitro. (a) Reduction in EZH2 transcript level after transfection with siRNA ( $40 \mathrm{nM}$ ) targeting EZH2 relative to cells transfected with scrambled siRNA $(40 \mathrm{nM})$, evaluated at $72 \mathrm{~h}$ post-transfection. The data are presented as mean \pm S.E. from two independent experiments, $n=6$; ${ }^{* \star \star} P \leq 0.0005$. (b) Western blot for EZH2 protein expression, also evaluated at $72 \mathrm{~h}$ post-transfection with siRNA targeting EZH2. (c) EZH2 depletion significantly reduced C666- 1 cell viability at days 4 and day 6 post-transfection, which is further enhanced by IR ( 4 Gy). The data are presented as mean \pm S.E. from two independent experiments, $n=6$; ${ }^{* \star \star} P \leq 0.0005 ;{ }^{* \star \star} P \leq 0.00005$. (d) Combination index analysis demonstrating more than additive interaction between EZH2 depletion and IR

NPC cells depleted of EZH2 exhibited delayed tumor growth in vivo. To assess whether targeting $\mathrm{EZH} 2$ has an effect in vivo, C666-1 cells were transfected with siEZH2 as single agent or combined with IR (4 Gy), before implanting into SCID mice. This resulted in a significant reduction in tumor formation in which no tumors were observed for 25 days in the siEZH2-treated cells, which was delayed until 31 days when siEZH2 depletion was combined with IR (Figure 3d), suggesting a potential therapeutic role for targeting $\mathrm{EZH} 2$.

Multiple pathways are affected by EZH2 depletion. To determine the pathways mediating cytotoxicity post-EZH2 depletion, with or without IR (4Gy), microarray analyses were conducted comparing C666-1 cells transfected with siEZH2 versus scrambled siRNA at $72 \mathrm{~h}$. Not surprisingly, several hundred genes were differentially expressed (up and down) post-siEZH2 transfection (Figure 4a, Supplementary Tables 1 and 2). The changes in expression of 10 selected transcripts were validated using individual quantitative realtime PCR (qRT-PCR) (Figure 4b). Pathway and functional annotation analyses performed on these differentially expressed genes in EZH2-depleted cells (both siEZH2 and siEZH2 plus IR) revealed significant enrichment for genes involved in cell differentiation, development, and apoptosis (Figure 4c). These data are consistent with our observed induction of apoptosis in EZH2-depleted cells (Figures $3 \mathrm{a}$ and $b$ ), and with the established roles for PcG genes in regulating cell differentiation and development. ${ }^{15}$ To confirm that these differentially expressed genes were indeed mediating the observed consequences of $\mathrm{EZH} 2$ knock down, BCL2, and FOXM1, which were down regulated upon $\mathrm{EZH} 2$ depletion, were individually targeted using siRNA. Following BCL2 and FOXM1 depletion in C666-1 cells, the same cell cycle changes of increased apoptosis, increased G1, and decreased populations in S-G2M were recapitulated as with siRNA targeting EZH2 (comparing Figure 4d with left panel of Figure 3a). Thus, these data corroborate that downregulation of BCL2 and FOXM1 participate in the cell cycle changes subsequent to siEZH2. Interestingly, when we looked for differentially expressed genes between siEZH2 plus $\mathrm{RT}$ and siEZH2 alone, we observed a cluster of genes, which is involved in the response to DNA damage and DNA repair (Supplementary Figure 2), thus supporting our conclusion of the involvement of EZH2 in this process. Other relevant pathways include those involved in response to oxidative stress (Supplementary Table 3); hence Trolox, a commonly utilized antioxidant was evaluated to determine whether it could rescue C666-1 cells from cell death post-EZH2 depletion. Indeed, pretreatment with Trolox led to a significant reduction in apoptosis following EZH2 depletion (Figure 4e), hence confirming that ROS were produced subsequent to EZH2 depletion, and thereby mediating cytotoxicity.

Identification of miR-26a, 98, and 101 as negative regulators of EZH2. The precise molecular mechanism(s) leading to $\mathrm{EZH} 2$ overexpression in NPC remains unknown. To focus on the role of miRs in this process, three commonly utilized miR target prediction algorithms (TargetScan, PicTar, 
a
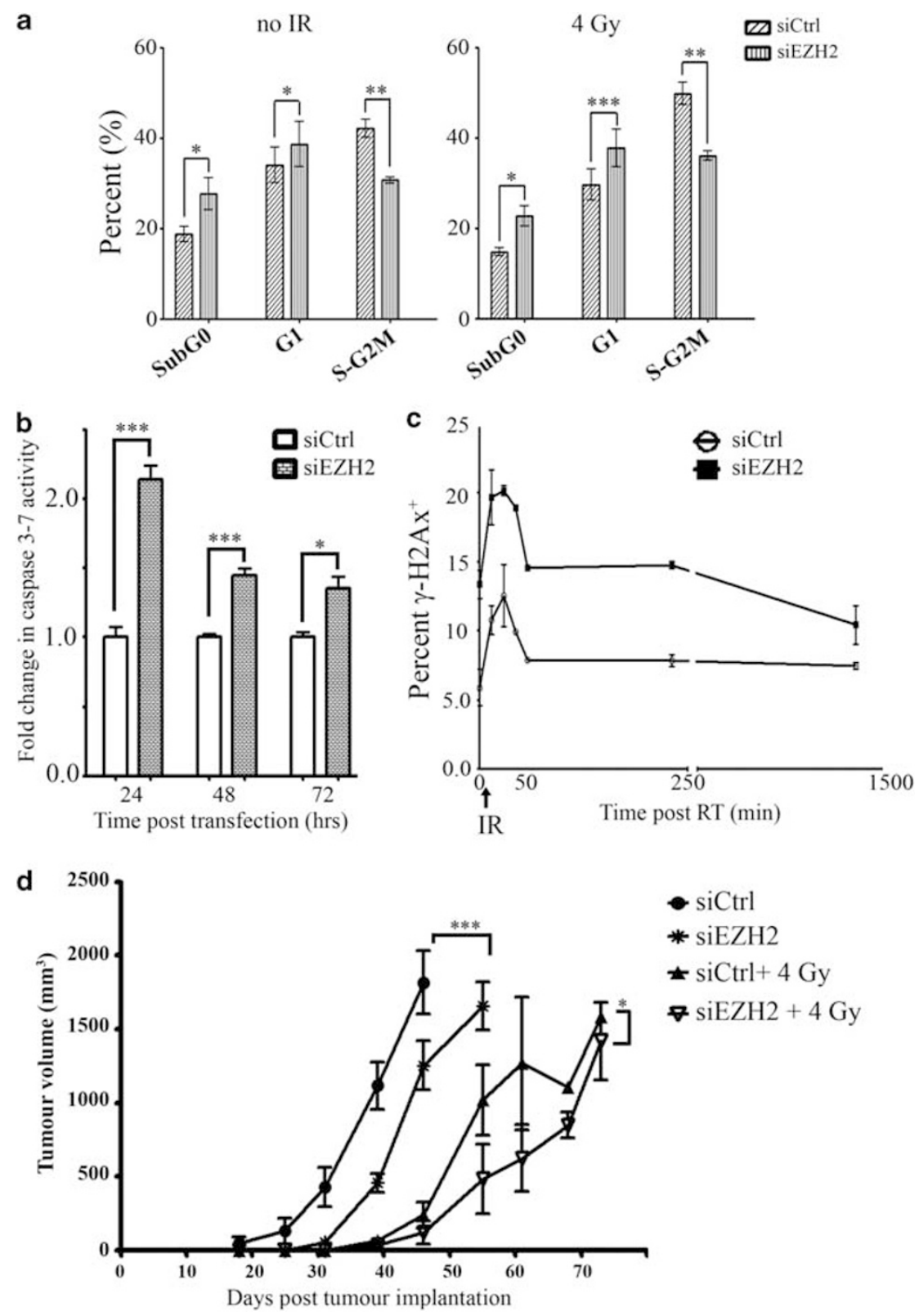

Figure 3 EZH2 depletion induced apoptosis in C666-1 cells and delayed tumor growth in vivo. (a) C666-1 cells were transfected with siCtrl ( $40 \mathrm{nM}$ ) or siEZH2 (40 nM), then exposed to IR (4 Gy) on day 3; cell cycle analyses were conducted on day 4 post-transfection. Data are presented as the mean \pm S.E. from two independent experiments, $n=6 ;{ }^{*} P \leq 0.05 ;{ }^{\star \star} P \leq 0.005 ;{ }^{* \star *} P \leq 0.0005$. (b) Caspase $3 / 7$ activity was measured at 24 , 48 , and $72 \mathrm{~h}$ post-transfection with the same siRNAs as in (a). Data are presented as the mean \pm S.E. from two independent experiments, $n=4 ;{ }^{*} P \leq 0.05 ;{ }^{* * *} P \leq 0.0005$. (c) C666-1 cells were transfected with the same siRNAs as in (a), then exposed to IR ( $4 \mathrm{~Gy}$ ) at $48 \mathrm{~h}$ post-transfection. The proportion of $\gamma-\mathrm{H} 2 \mathrm{AX}$ expressing cells was measured at the indicated time points. Data are presented as the mean $\pm \mathrm{S}$.E. from two independent experiments, $n=2$. (d) C666-1 cells were transfected with siCtrl ( $40 \mathrm{nM})$, or siEZH2 ( $40 \mathrm{nM}$ ) for $72 \mathrm{~h}$, then exposed to IR (4 Gy), followed by implantation in SCID mice on day 6 post-transfection. Tumor growth was then monitored over time. The data are presented as mean \pm S.E. for each group of mice, each group consisting of 6 mice. Two-way ANOVA analysis was utilized to compare the difference in tumor growth between the indicated groups. ${ }^{*} P \leq 0.05 ;{ }^{* * \star} P \leq 0.0005$

and MiRanda) were interrogated for possible interactions with $\mathrm{EZH} 2$. The intersection of these three algorithms identified six potential miR candidates: miR-101, 138, 217, 26a, 26b, and 98 (Figure 5a). The expression of these six miRs was then evaluated in 15 human NPC biopsy specimens (cohort ' $B$ '), demonstrating that miR-26a, 26b, and 101 were all consistently downregulated, compared with normal nasopharyngeal epithelial tissues, although their expression levels did not associate with clinical outcome
(Figure 5b). Interestingly however, miR-98 was significantly underexpressed in relapsed versus nonrelapsed NPC samples $(P=0.05)$; hence, these four miRs (miR-26a, 26b, 101 , and miR-98) were examined in further detail.

Exogenous expression of miR-26a, 98, and 101 caused cytotoxicity associated with downregulation of EZH2. We then sought to determine whether overexpressing these four miRs could mimic the phenotypic changes observed with 
a
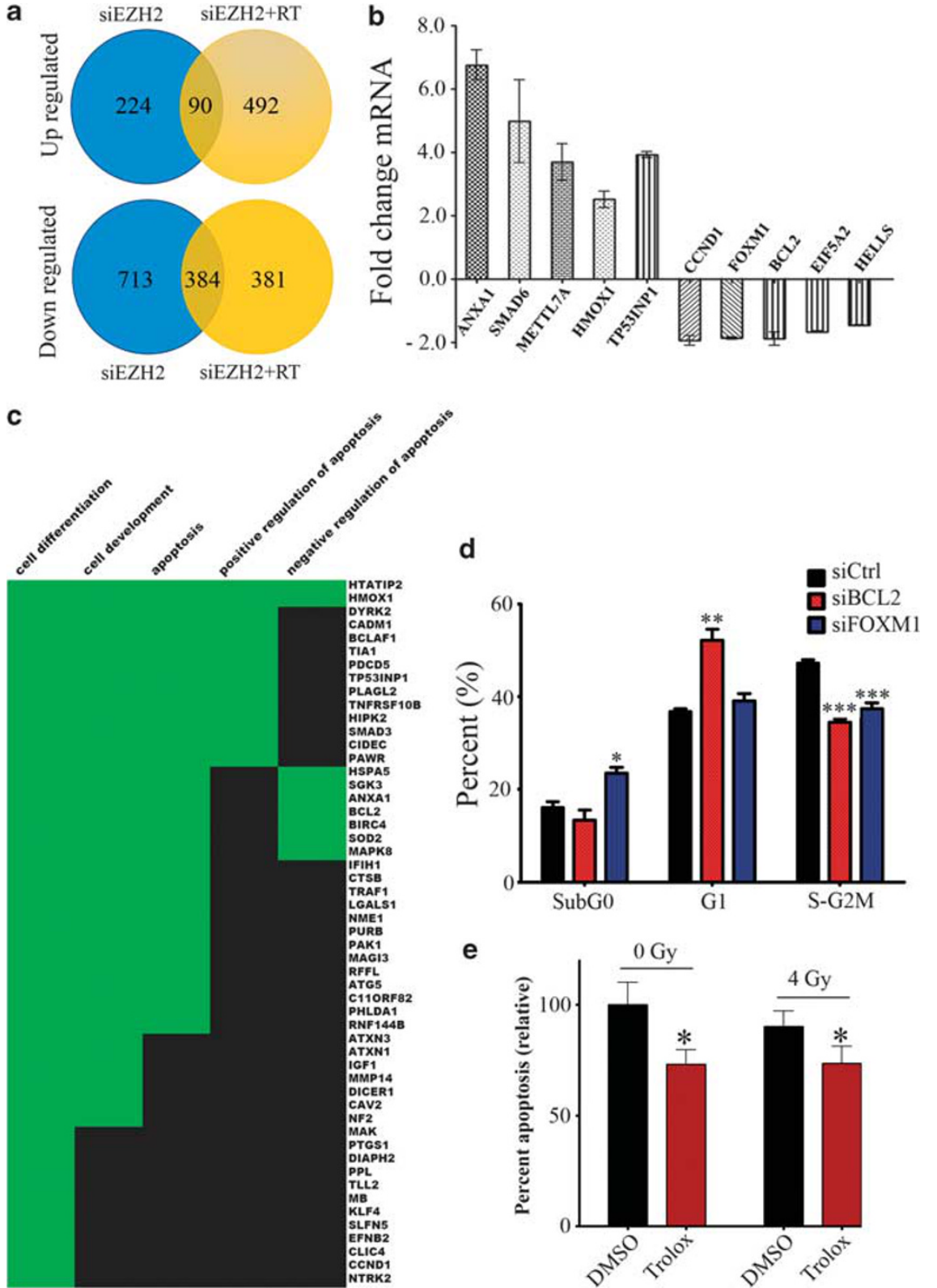

e

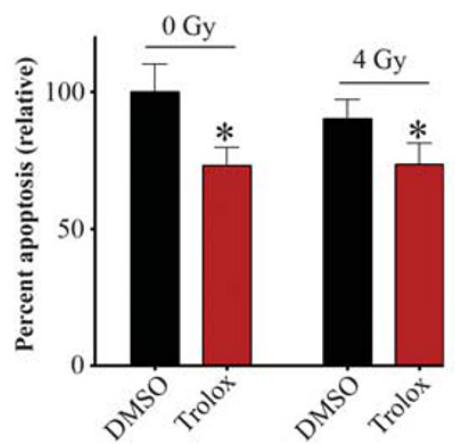

Figure 4 Genome-wide microarray and pathway analyses reveal multiple pathways regulated by EZH2 in NPC. (a) Venn diagram showing the number of differentially expressed genes in cells depleted of EZH2 in the presence or absence of IR. C666-1 cells were transfected with siCtrl (40 nM) or siEZH2 ( $40 \mathrm{nM}$ ) for $48 \mathrm{~h}$, then exposed to IR (4 Gy). RNA was extracted at $72 \mathrm{~h}$ post-transfection, then hybridized onto the Human Genome U133 Plus 2.0 Array. (b) A representative set of 10 differentially expressed genes validated using individual qRT-PCR, performed at $72 \mathrm{~h}$ post-transfection with siEZH2 $(40 \mathrm{nM})$, compared with cells transfected with siCtrl ( $40 \mathrm{nM})$. Data are presented as mean \pm S.E. from two independent experiments, $n=6$. (c) Heatmap displaying enrichment for genes involved in different cellular processes in EZH2-depleted cells; green color denotes association; black color denotes absence of association with the indicated cellular processes. (d) C666-1 cells were transfected with $40 \mathrm{nM}$ of siCtrl, siBLC2, or siFOXM1, then cell cycle analyses were conducted at $72 \mathrm{~h}$ post-transfection. The proportion of cells in each stage of the cell cycle is indicated. Data are presented as mean \pm S.E. from two independent experiments compared with cells transfected with siCtrl, $n=4 ;{ }^{*} P \leq 0.05 ;{ }^{* \star} P \leq 0.005 ;{ }^{* \star \star} P \leq 0.0005$. (e) C666-1 cells were transfected with siEZH2 (40 nM), then treated with Trolox (500 $\mu \mathrm{M}$ on day 1 and $500 \mu \mathrm{M}$ on day 2). At $2 \mathrm{~h}$ after second Trolox treatment, half of the cells were then exposed to $4 \mathrm{~Gy}$ IR, and the percent of apoptosis was measured using flow cytometry on day 3 . The data are presented as mean \pm S.E. from two independent experiments, compared with DMSOtreated conditions, $n=4 ;{ }^{*} P \leq 0.05$

EZH2 depletion. Exogenous expression of miR-26a, 98, and 101 all led to significant toxicity in C666-1 cells, which increased with the addition of IR, whereas overexpression of miR-26b had no effect (Figure 5c). To further determine whether the three remaining miRs were regulating EZH2, qRT-PCR was utilized to measure EZH2 mRNA expression after transfection with miR-26a, 98, and 101. Indeed, significant reduction in EZH2 transcript levels was observed for all three miRs (Figure 5d). miR-101 has previously been described to regulate EZH2 expression in prostate cancer; ${ }^{16}$ hence, we sought to determine whether the two novel miRs (miR-26a and miR-98, which have not been 

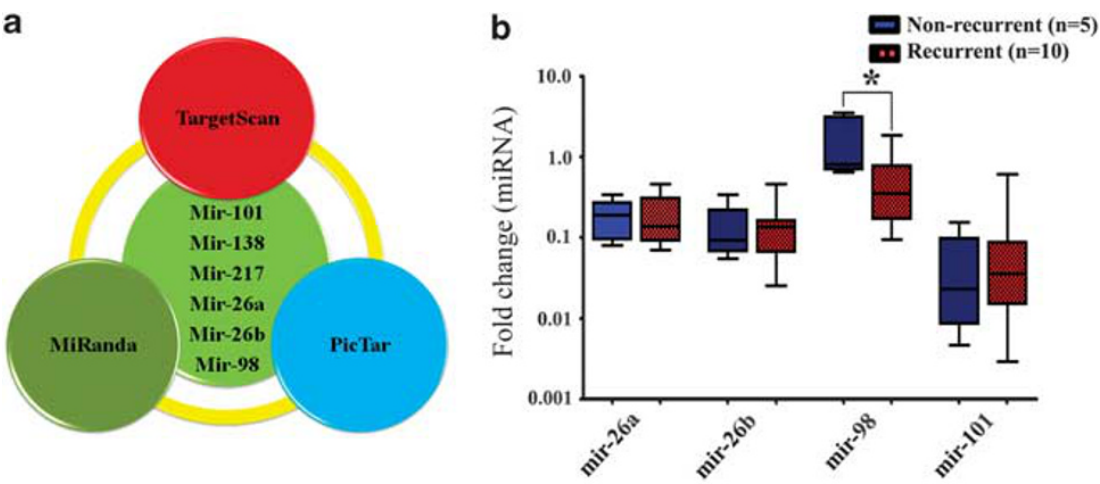

C
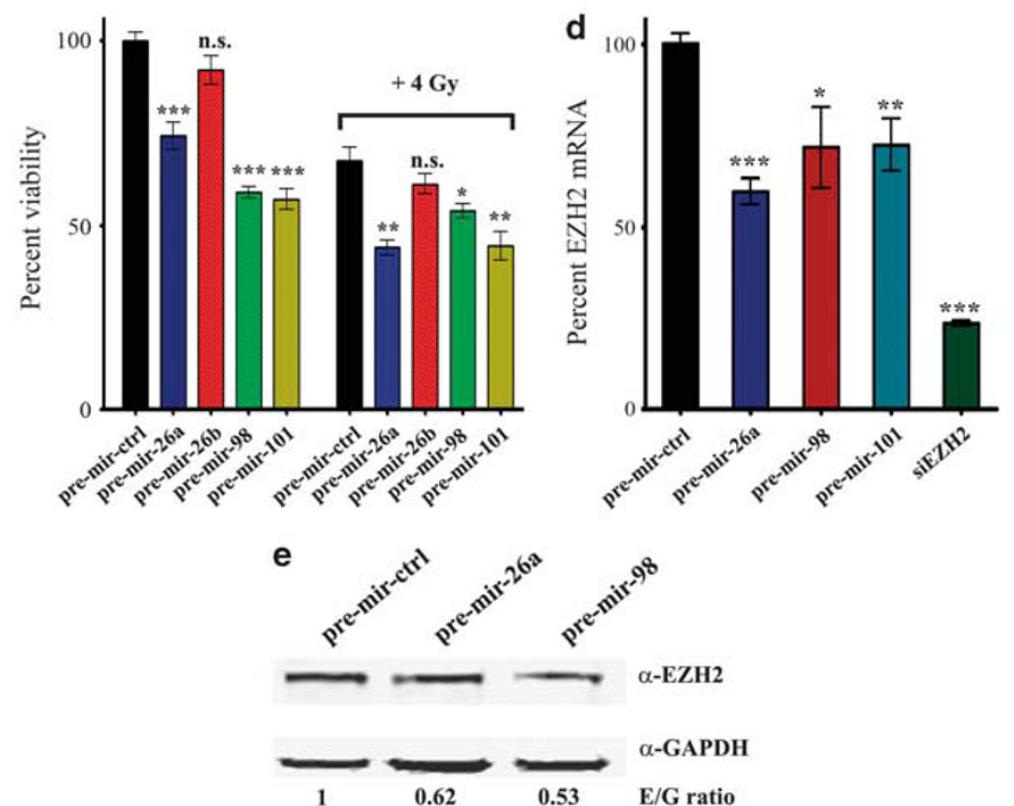

Figure 5 Negative regulation of EZH2 expression by miR-26a, 98, and 101. (a) Venn diagram showing the miRs that were predicted by all three databases (TargetScan, MiRanda, and PicTar), which can potentially regulate EZH2. (b) Fold change in miR-26a, 26b, 98, and 101 expression in 10 recurrent and 6 nonrecurrent primary NPC specimens compared with normal nasopharyngeal epithelial tissues (normalized to 1, on a log scale), evaluated in cohort 'B'. (c) MTS viability for C666-1 cells on day 6 posttransfection with the indicated miRs $(40 \mathrm{nM})$. IR (4 Gy) was delivered $24 \mathrm{~h}$ post-transfection. The data are presented as mean \pm S.E. from two independent experiments, $n=8$; ${ }^{*} P \leq 0.05 ;{ }^{* *} P \leq 0.005 ;{ }^{* \star *} P \leq 0.0005$. (d) qRT-PCR analysis of the fold-change in EZH2 mRNA expression 72 hrs post-transfection with the indicated miRs (all using $40 \mathrm{nM}$ ). Data are presented as mean \pm S.E. from two independent experiments, $n=6 ;{ }^{*} P \leq 0.05 ;{ }^{* *} P \leq 0.005 ;{ }^{* *} P \leq 0.0005$. (e) Western blot analysis for EZH2 expression in $\mathrm{C} 666-1$ cells at $72 \mathrm{~h}$ post-transfection with the indicated pre-miRs; $\mathrm{E} / \mathrm{G}$ : ratio of EZH2/GAPDH

previously described to regulate $\mathrm{EZH} 2$ in solid tumors) regulated $\mathrm{EZH} 2$ at the protein level. As shown in Figure $5 \mathrm{e}$, overexpression of miR-26a or miR-98 reduced EZH2 protein expression, corroborating the biological effects of these two miRs in regulating EZH2 in NPC. Nonetheless, an inverse relationship between miR-98, miR-26a, and EZH2 expression was observed in the FaDu and UTSCC-8 cell lines (Supplementary Figure 3). Knocking down miR-26a only led to significant increase in EZH2 expression in the FaDu cell line (Supplementary Figure 4), presumably because of its higher basal level expression in those cells.

The $3^{\prime}$-UTR of EZH2 transcript is a direct target for miR-26a, 98. To demonstrate the direction interaction of miR-26a or miR-98 with EZH2, the $3^{\prime}-\mathrm{UTR}$ of EZH2 was cloned downstream of the firefly luciferase gene in the
pMIR-REPORT vector (with the predicted binding sites for the miR-26a and miR-98 indicated (Figure 6a)). miR-26a, or miR-98 were individually co-transfected with pMir-REPORTEZH2 UTR, leading to significant reduction in luciferase activity compared with cells transfected with control miR or pMir-REPORT control vector (Figure 6b). Mutating the seed region for miR-26a and miR-98 binding in the pMIR-REPORT-EZH2 UTR vector completely abrogated its regulatory activity (Figure $6 \mathrm{~b}$ ), thereby confirming that miR-26a and miR-98 directly interacted with the $3^{\prime}$-UTR of $\mathrm{EZH} 2$. We then sought to determine the relative expression levels of EZH2 and miR-98 in an independent collection of NPC samples (cohort ' $C$ '). It was gratifying to observe an inverse relationship between EZH2 and miR-98 expression (Figure 6c), thereby supporting the notion of $\mathrm{EZH} 2$ regulation by miR-98 in NPC, although such relationship was less observed at the single specimen level. 
a

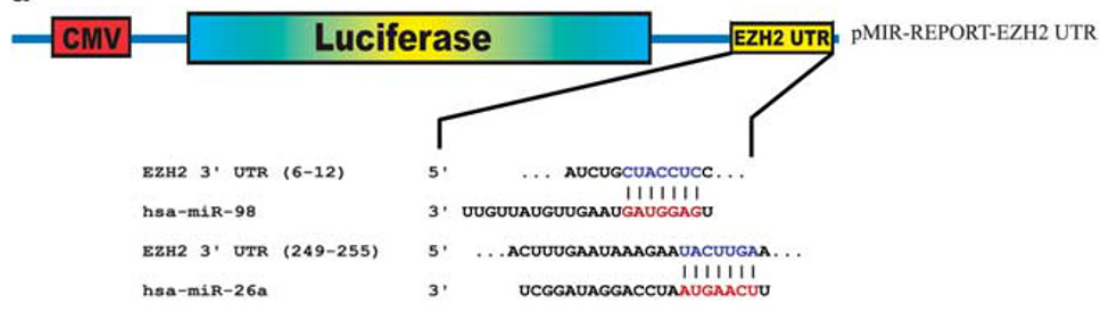

b

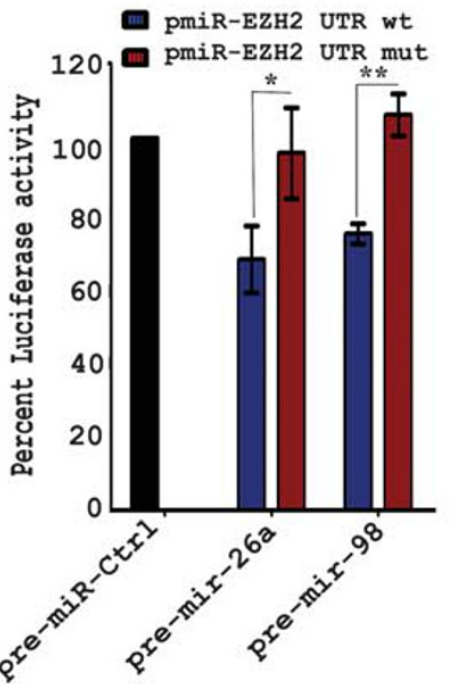

C

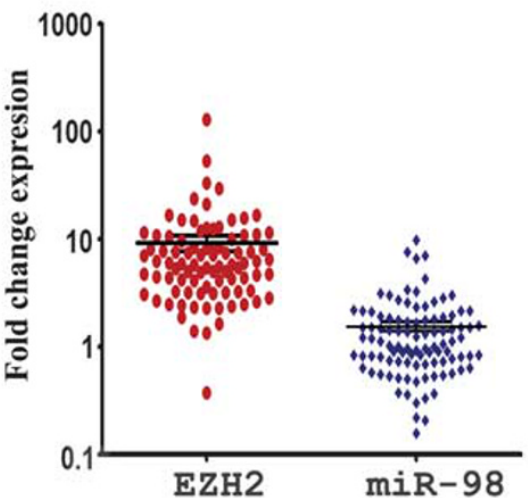

d

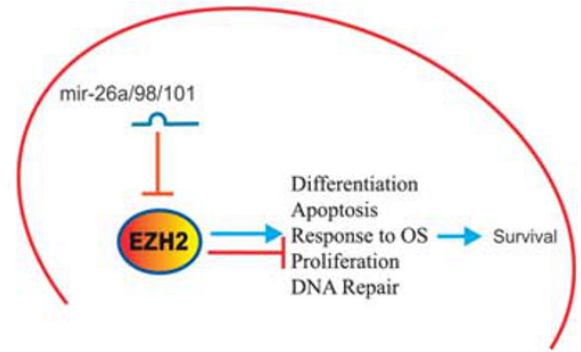

Figure 6 EZH2 $3^{\prime}$-UTR is a direct target of miR-26a, 98, and 101. (a) Schematic representation of the pMIR-REPORT-EZH2 UTR expression vector with the alignment of the indicated miRs with the EZH2 $3^{\prime}$-UTR with the seed region highlighted in blue (miR), and red (UTR). (b) Percent luciferase activity at $48 \mathrm{~h}$ post-transfection with the indicated miR $(100 \mathrm{nM})$, and reporter plasmid $(100 \mathrm{ng})$. Data are presented as mean \pm S.E. from two independent experiments, $n=4$; ${ }^{*} P \leq 0.05$; ${ }^{* \star} P \leq 0.005$. (c) Inverse expression of EZH2 $(n=89)$ with miR-98 $(n=99)$ is observed in primary NPC specimens (cohort 'C'), as measured by qRT-PCR and presented as fold-change expression in tumors compared with normal nasopharyngeal epithelial tissues $(n=6) .{ }^{* \star} P \leq 0.0005$. (d) A proposed model derived from these data, illustrating that underexpression of miR-26a, 98, and 101 lead to upregulation of EZH2, which in turn mediates several important cellular processes all driving the survival of NPC cells; (OS: oxidative stress)

\section{Discussion}

Although several reports in the literature documented overexpression of $\mathrm{EZH} 2$ in human malignancies, the precise role of $\mathrm{EZH} 2$ in tumorigenesis and the molecular mechanisms leading to its overexpression remain largely unknown. In this study, a comprehensive analysis of EZH2 was conducted, starting with its potential clinical value in NPC, determining the cellular processes regulated by this gene, and documenting a mechanism leading to its overexpression. The in vitro experiments demonstrated that $\mathrm{EZH} 2$ promote survival of NPC cells in that its targeted depletion led to cytotoxicity, mediated by apoptosis and delayed progression through the cell cycle, translating into delayed tumor growth in vivo (Figure 3). These data are consistent with reports in the literature describing similar effects of EZH2 depletion in other cancer models. $^{17,18}$

EZH2 is located on chr7q36.1, which is not a common site of chromosomal gain in NPC. ${ }^{19}$ Thus, alternate mechanism(s) accounting for $\mathrm{EZH} 2$ overexpression was sought, focusing on miRs, which are increasingly recognized as a major class of regulatory molecules, by targeting mRNAs for degradation, and inhibiting protein translation. Using a combinatorial approach of miR expression profiling, bioinformatics, and biochemical tools, we demonstrated that miR-26a, 98, and 101 , which are also underexpressed in NPC, could negatively regulate $\mathrm{EZH} 2$ (Figures $5 \mathrm{~b}$ and 6 ). These findings corroborate recent reports of miR-26a in regulating EZH2 in Burkitt's lymphoma, ${ }^{20}$ and $\mathrm{miR}-101$ targeting $\mathrm{EZH} 2$ in prostate cancer. ${ }^{16}$ In NPC, however, at least three miRs are shown 
here to target EZH2; specifically, miR-98 is a novel negative regulator of $\mathrm{EZH} 2$, whose functional significance is underscored by its downregulation in recurrent NPC (Figure 5b), consistent with overexpression of EZH2 associating with poor survival (Figure 1b and c).

Genome-wide microarray analyses revealed that several hundred genes are either up- or downregulated following $\mathrm{EZH} 2$ depletion, suggesting a dual role for $\mathrm{EZH} 2$ in both silencing and activating transcription (Figure $4 \mathrm{a}$ and Supplementary Tables 1 and 2). The latter might appear paradoxical given that PcG genes are normally viewed as mediators of gene repression; however, there is increasing evidence that PcG genes can also mediate transcription, possibly through long-distance chromosomal interactions. ${ }^{17,21}$ Among the downregulated genes following $\mathrm{EZH} 2$ depletion were FOXM1 and BCL2, which we had previously reported to be upregulated in primary biopsy samples of NPC, ${ }^{12}$ which one might speculate could relate to EZH2. Functional analyses documented that FOXM1 and BCL2 indeed mediated the phenotypic changes of apoptosis and cell cycle changes, mimicking the same alterations as observed in EZH2-depleted cells.

Multiple other processes are also affected by EZH2, such as cellular response to oxidative stress, in which pretreatment of C666-1 cells with the antioxidant Trolox protected against cell death induced by EZH2 depletion (Figure $4 \mathrm{e}$ ). A related observation has been reported by both ourselves, and others, whereby BMI-1, another member of the PcG family, has a role in maintaining ROS homeostasis in both cancer and normal cells). ${ }^{11,22,23}$ Hence, it would appear that PcG genes, such as BMI1 and EZH2, participate in ROS regulation in mammalian cells.

In conclusion, our data are the first to document that EZH2 expression could serve as a potential prognostic marker for NPC, and its overexpression mediates several important cellular processes, all resulting in promoting survival (see Figure 6d). In addition, miR-26a, 98, and 101 can all regulate EZH2 expression, with miR-98 perhaps being its most functionally significant negative regulator.

\footnotetext{
Materials and Methods

Patient samples. In total, 39 formalin-fixed and paraffin-embedded (FFPE) NPC patient biopsy samples were selected for immunohistochemical analysis of EZH2 from an archival bank of materials as previously described (cohort ' $A$ '). ${ }^{24}$ These patients have all been treated with curative intent, using IR for early-stage disease, or combined with chemotherapy for locally advanced disease, at the Princess Margaret Hospital (PMH) during the period of 1985-1992. Owing to insufficient tissue materials from cohort ' $A$ ', global miR expression profiling was conducted on 15 additional NPC samples treated similarly at PMH during the period of 1993-2000 (cohort 'B'). A third cohort ' $C$ ' was then utilized to examine the correlation between EZH2 and miR-98 expression. All tissues utilized in this study have received institutional REB approval.
}

Immunohistochemical detection of EZH2 expression. EZH2 immunohistochemistry analysis was evaluated on 4- $\mu \mathrm{m}$ FFPE tumor sections using microwave antigen retrieval, in combination with the LSAB + System-HRP (Dako North America, Carpinteria, CA, USA). Detection of EZH2 utilized rabbit antiEZH2 antibody (dilution 1/1000; Invitrogen, Corp., Carlsbad, CA, USA). EZH2 expression was scored using a previously published protocol for EGFR, ${ }^{25}$ in which a score was assigned by combining the percentage of cells with positive EZH2 staining $(0-5)$, with the intensity of staining $(1+$ to $3+)$. Any multiplied values $\geq 10$ were scored as high EZH2 expression.
Quantitative real-time PCR. QRT-PCR analyses were performed using the ABI PRISM 7900 Sequence Detection System (Applied Biosystems Inc., Foster City, CA, USA). The primer sequences used in this study are listed in Table 1. To assess the efficiency of EZH2 knockdown, C666-1 cells were transfected with $40 \mathrm{nM}$ siEZH2 or siCtrl, and at indicated time points, cells were collected, and total RNA was isolated using the RNeasy Mini Kit (Qiagen, Chatsworth, CA, USA). Reverse transcription was performed using SuperScript III Reverse Transcriptase (Invitrogen Corp.) according to the manufacturer's recommendations. The relative fold change in RNA expression was calculated using the $2^{-\Delta \Delta C}$ method, where the average of $\triangle C t$ values for the amplicon of interest was normalized to that of an endogenous gene (GAPDH), compared with control specimens. Global miR expression and single-well miR PCR were conducted as previously described. ${ }^{26}$

Cell culture and transfection. The EBV-positive C666-1 NPC cell line ${ }^{27}$ was maintained in RPMl 1640 supplemented with $10 \%$ fetal bovine serum, $100 \mathrm{mg} / \mathrm{l}$ penicillin, and $100 \mathrm{mg} / \mathrm{l}$ streptomycin (RPMI- 10 ) at $37^{\circ} \mathrm{C}$ and $5 \% \mathrm{CO}_{2}$. SMARTpool siRNAs targeting EZH2, EED, BCL2, and FOXM1 were purchased from Dharmacon (Dharmacon Inc., Lafayette, CO, USA). Control siRNAs were purchased from either Dharmacon or Ambion (Ambion, Austin, TX, USA). miR precursors (pre-miR) were purchased from Ambion. Locked nucleic acid probes containing sequence-specific antisense oligonucleotides targeting miR-26a and miR-98 were purchased from Exiqon (Exiqon Inc., Vedbaek, Denmark). C666-1 cells were transfected with the indicated siRNA or pre-miR using the Lipofectamine RNAiMAX (Invitrogen) reverse transfection protocol, according to the manufacturer's instructions. At 48 or $72 \mathrm{~h}$ post-transfection, cells were exposed to IR (4 Gy).

Immunoblotting . C666-1 cells were transfected with $40 \mathrm{nM}$ siEZH2, siCtrl, pre-miR-26a, pre-miR-98, or pre-miR-Ctrl; at $72 \mathrm{~h}$ post-transfection, cells were collected and lysed in $1 \mathrm{M}$ Tris- $\mathrm{HCl}(\mathrm{pH} 8), 5 \mathrm{M} \mathrm{NaCl}$, and $1 \% \mathrm{NP} 40$ plus protease inhibitor cocktail (Roche Diagnostics, Quebec, Canada). Protein concentration was determined using the Bio-Rad Detergent-Compatible Protein Assay (Bio-Rad Laboratories, Hercules, CA, USA). In total, $20 \mu \mathrm{g}$ of protein were loaded onto $10 \%$ Tris-glycine protein gels (Invitrogen) for electrophoresis. The protein was then transferred onto a nitrocellulose membrane using a Trans-Blot SD Semi-Dry Transfer Cell (Bio-Rad) and blocked using TBST (0.1\% Tween-20 plus 5\% fat-free dry milk). The membrane was probed with anti-EZH2 monoclonal antibody (clone AC22, 1:1000 dilution, Cell Signaling Technology Inc., Danvers, MA, USA) and anti-GAPDH antibody (Abcam, Cambridge, MA, USA).

Cell viability and measurement of apoptosis. Cell viability was assessed using the soluble tetrazolium salt (MTS) cell proliferation assay as previously described, ${ }^{28}$ as these cells are not amenable to clonogenic assays. Briefly, $20 \mu$ of the MTS reagent (Promega, Madison, WI, USA) was added to each well in a 96-well plate at the indicated time points, and absorbance was measured at $\lambda_{492}$. To measure the fraction of cells in the sub G0-G1 phase of the cell cycle, cells were collected and washed twice in FACS buffer (PBS/0.5\% BSA). Cells were resuspended in $1 \mathrm{ml}$ of FACS buffer, and then $3 \mathrm{ml}$ of ice-cold $70 \%$ ethanol was added to fix the cells for $1 \mathrm{~h}$ on ice. Cells were washed once, before resuspending in $500 \mu$ l of FACS buffer containing $40 \mu \mathrm{g} / \mathrm{ml}$ RNAse A (Sigma, St. Louis, MO, USA) and $50 \mu \mathrm{g} / \mathrm{ml}$ propidium iodine. Cells were incubated at room temperature for $30 \mathrm{~min}$ in the dark before being analyzed in BD FACScalibur (Becton Dickinson, San Jose, CA, USA) using FL-2A and FL-2W channels. FACS data were analyzed using FlowJo 7.5 software (Tree Star, San Carlos, CA, USA).

Caspase activation was measured using Promega Apo-ONE Homogeneous Caspase-3/7 assay. Briefly, cells were transfected with siCtrl or siEZH2, and $48 \mathrm{~h}$ later, exposed to 4 Gy IR. At indicated time points, caspase $3 / 7$ substrates were added according to the manufacturer's specifications. Plates were analyzed using SPECTRAFluor Plus Fluorometer (Tecan, Research Triangle Park, NC, USA) at excitation and emission wavelengths of 485 and $535 \mathrm{~nm}$, respectively.

Quantitation of phosphorylated H2AX ( $\gamma$-H2AX). A total of 50000 C666-1 cells were transfected with $40 \mathrm{nM}$ siEZH2 or siCtrl, and $48 \mathrm{~h}$ later, exposed to IR (4 Gy). Staining for $\gamma$-H2AX was conducted as previously described. ${ }^{11}$ Both attached and detached C666-1 cells were collected at the indicated time points post-IR, pelleted by centrifugation, washed in FACS buffer (PBS/0.5\% BSA), fixed with $3 \mathrm{ml} 70 \%$ ethanol, and maintained at $-20^{\circ} \mathrm{C}$. After collecting all samples, cells were pelleted by centrifugation, and rehydrated with $1 \mathrm{ml}$ cold TBS ( $\mathrm{pH}$ 7.4) plus 4\% FBS, and $0.1 \%$ Triton X-100 (TST) on ice for $10 \mathrm{~min}$. Cells were then centrifuged and resuspended in $200 \mu \mathrm{l}$ of FITC-labeled mouse monoclonal antiphosphorylated 
Table 1 Primer sequences used for cloning and qRT-PCR

\begin{tabular}{|c|c|c|}
\hline No. & Name & Sequence \\
\hline 1 & $\begin{array}{c}\text { EZH2 } \\
\text { FWD } \\
\text { REV }\end{array}$ & $\begin{array}{l}\text { 5'-GCGCGGGACGAAGAATAATCAT-3' } \\
\text { 5'-TACACGCTTCCGCCAACAAACT-3' }\end{array}$ \\
\hline 2 & $\begin{array}{c}A N X A 1 \\
\text { FWD } \\
\text { REV }\end{array}$ & $\begin{array}{l}\text { 5'-GAGATTTTCGGAACGCTTTG-3' } \\
\text { 5'-GCTTCTGGTGGTAAGGATGG-3' }\end{array}$ \\
\hline 3 & $\begin{array}{c}\text { SMAD6 } \\
\text { FWD } \\
\text { REV }\end{array}$ & $\begin{array}{l}\text { 5'-TGCAACCCCTACCACTTCA-3' } \\
\text { 5'-ATTCACCCGGAGCAGTGAT-3' }\end{array}$ \\
\hline 4 & $\begin{array}{l}\text { METTL7A } \\
\text { FWD } \\
\text { REV }\end{array}$ & $\begin{array}{l}\text { 5'-CTGATGGCTCTGTGGATGTG-3' } \\
\text { 5'-TTCCAAGTCGAACACTCAGC-3' }\end{array}$ \\
\hline 5 & $\begin{array}{l}\text { HMOX1 } \\
\text { FWD } \\
\text { REV }\end{array}$ & $\begin{array}{l}\text { 5'-CCAGGCAGAGAATGCTGAGT-3' } \\
\text { 5'-GTAGACAGGGGCGAAGACTG-3' }\end{array}$ \\
\hline 6 & $\begin{array}{l}\text { TP53INP1 } \\
\text { FWD } \\
\text { REV }\end{array}$ & $\begin{array}{l}\text { 5'-CCTCCAACCAAGAACCAGAA-3' } \\
\text { 5'-TGCCGGTAAACAGGAAAAGA-3' }^{\prime}\end{array}$ \\
\hline 7 & $\begin{array}{c}\text { CCND1 } \\
\text { FWD } \\
\text { REV }\end{array}$ & $\begin{array}{l}\text { 5'-CGTGGCCTCTAAGATGAAGG-3' } \\
5^{\prime} \text {-CTGGCATTTTGGAGAGGAAG-3' }\end{array}$ \\
\hline 8 & $\begin{array}{c}\text { FOXM1 } \\
\text { FWD } \\
\text { REV }\end{array}$ & $\begin{array}{l}\text { 5'-AACCTGCAGCTAGGGATGTG-3' } \\
\text { 5'-AGCCCAGTCCATCAGAACTC-3' }\end{array}$ \\
\hline 9 & $\begin{array}{c}\text { BCL2 } \\
\text { FWD } \\
\text { REV }\end{array}$ & $\begin{array}{l}\text { 5'-GAGTTCGGTGGGGTCATGT-3' } \\
\text { 5'-ACAGTTCCACAAAGGCATCC-3' }\end{array}$ \\
\hline 10 & $\begin{array}{c}\text { EIF5A2 } \\
\text { FWD } \\
\text { REV }\end{array}$ & $\begin{array}{l}\text { 5'-TCCAGCACTTACCCTATGCAG-3' } \\
\text { 5'-TGCCCGTGAAAATATCAATTC-3' }\end{array}$ \\
\hline 11 & $\begin{array}{c}\text { HELLS } \\
\text { FWD } \\
\text { REV }\end{array}$ & $\begin{array}{l}\text { 5'-GCAATGGTTGAACAACTGGA-3' } \\
\text { 5'-ACGGTACCGAATTTCTGTCG-3' }\end{array}$ \\
\hline 12 & $\begin{array}{l}\text { EZH2 } \\
\text { UTR FWD } \\
\text { UTR REV }\end{array}$ & $\begin{array}{l}\text { 5'-GTTGTTACTAGTCATCTGCTACCTCCTCC-3' } \\
\text { 5'-GTTGTTAAGCTTACAAGTTCAAGTATTCTTTATTC-3' }\end{array}$ \\
\hline 13 & $\begin{array}{l}\text { EZH2 } \\
\text { UTR REV miR-26a MUT }\end{array}$ & 5'-GTTGTTAAGCTTACAGAGGAGGGTATTCTTTATTCAAA-3' \\
\hline 14 & $\begin{array}{l}\text { EZH2 } \\
\text { UTR FWD miR-98 MUT }\end{array}$ & $\begin{array}{l}\text { 5'-GTTGTTACTAGT CATCTGACCAACAATCCC CCCTCCTCTGAAC } \\
\text { ACAACGC CTTAAACCAC GGAACCTCGA GTACTGTGGG-3' }\end{array}$ \\
\hline
\end{tabular}

(Ser139) histone H2AX antibody (1:500 dilution in TST, clone JBW301, Upstate Biotechnology Inc., Lake Placid, NY, USA) for $2 \mathrm{~h}$ at room temperature. Cells were then washed twice in TBS and analyzed using BD FACScalibur.

Tumor formation. All animal experiments were conducted in accordance with the guidelines of the Animal Care Committee, University Health Network. For tumor formation assays, $5 \times 10^{4}$ C666-1 cells were transfected with $40 \mathrm{nM}$ siEZH2 or siCtrl. At $72 \mathrm{~h}$ later, where indicated, cells were exposed to $4 \mathrm{~Gy}$ IR using a ${ }^{137} \mathrm{Cs}$ unit (Gamma-cell 40 Extractor; Nordion International, Inc., Ontario, Canada) at a dose rate of 1.1 Gy per min. On day 6 , cells were collected, resuspended in $100 \mu$ PBS, then injected intramuscularly into the left gastrocnemius of 6-8-week-old female SCID mice. Tumor volume was calculated as described before ${ }^{29}$ using the formula $\left(v=\pi \times \mathrm{d}^{3} / 6\right)$, where $\mathrm{d}$ is the diameter of leg plus tumor and volumes were corrected by subtracting the volume of nontumor-bearing leg. Mice were killed when the humane end point was reached.
Microarray and bioinformatics analyses. C666-1 cells were transfected with $40 \mathrm{nM}$ siEZH2 or siCtrl, and at $48 \mathrm{~h}$ post-transfection half of the transfected cells were exposed to IR ( $4 \mathrm{~Gy}$ ). Total RNA was extracted from five transfection replicates of irradiated and nonirradiated cells $17 \mathrm{~h}$ post-IR using the Qiagen RNeasy kit (Qiagen) according to the manufacturer's instruction, and tested for quality using the Agilent 2100 Bioanalyzer (Agilent Technologies Inc., Palo Alto, CA, USA). Extracted RNA was labeled and hybridized onto the Affymetrix GeneChip Human Genome U133 Plus 2.0 array (Affymetrix, Inc., Santa Clara, CA, USA). All procedures were conducted at the University Health Network Microarray center (Toronto, ON, Canada). The raw data were normalized using the GCRMA package of the Bioconductor open source library ${ }^{30}$ for $R$ (version 2.7.1) software. Only genes with 'present' calls demonstrating a $\geq$ two-fold change in expression over lipofectamine and siCtrl-transfected cells were included in the analysis. Heatmaps were generated using Microsoft excel 2007 (Microsoft, Inc., Redmond, WA, USA). Gene overlap was assessed using the gene list Venn diagram tool 
(http://mcbc.usm.edu/genevenn/genevenn.htm) Pathway analyses of differentially expressed genes were performed using DAVID Bioinformatics Database functionalannotation tools (http://david.abcc.ncifcrf.gov/). ${ }^{31}$

Inhibition of reactive oxygen species. C666-1 cells were transfected as described above, and at 24 and $48 \mathrm{~h}$ post-transfection, the antioxidant Trolox (Sigma) was added at $500 \mu \mathrm{M}$ concentration (from stock solution of $500 \mathrm{mM}$ in DMSO). At $2 \mathrm{~h}$ following the second Trolox treatment on day 2, cells were exposed to 4 Gy IR. Cells were collected on day 3, fixed in $70 \%$ ethanol, then stained and analyzed to measure the apoptotic fraction as described above.

Luciferase assay. EZH2 $3^{\prime}$-UTR region was amplified using the indicated primers in Table 1, and using AmpliTaq gold DNA polymerase (Applied Biosystems). The PCR product was then purified, digested with Spel and HindllI, then cloned downstream of the firefly luciferase gene in the PMIR-REPORT vector (Ambion). A separate vector carrying EZH2 $3^{\prime}$-UTR with mutation in the seed region for miR-26a or miR-98 was also constructed using the indicated primers in Table 1. At $72 \mathrm{~h}$ before transfection, $1 \times 10^{5} \mathrm{C} 666-1$ cells were seeded in a 24-well plate. Cells were then transfected with $100 \mathrm{nM}$ of pre-miR-ctrl, 26a, 98 , or 101 , and $6 \mathrm{~h}$ later, cells were co-transfected with $100 \mathrm{ng}$ of pMIR-REPORT or pMIR-REPORT EZH2 $3^{\prime}$-UTR, along with $50 \mathrm{ng}$ of pRL-TK vector (Promega) carrying the Renilla luciferase gene. Transfection experiments were conducted using Lipofectamine 2000 (Invitrogen). At $48 \mathrm{~h}$ later, luciferase activity was measured using the Dual-Glo luciferase assay system (Promega). Firefly luciferase activity was then normalized to that of Renilla luciferase.

Statistical analysis. Statistical analyses and graphing were performed using Microsoft excel and GraphPad Prism software (GraphPad software, San Diego, CA, USA), respectively. Outliers were removed from data using the extreme studentized deviate method (http://www.graphpad.com/quickcalcs/Grubbs1.cfm).

\section{Conflict of interest}

The authors declare no conflict of interest.

Acknowledgements. This work has been supported by funds from the Canadian Institutes of Health Research, and by Dr. Mariano Elia Chair in Head and Neck Cancer Research. We acknowledge the philanthropic support from the Wharton Family, Joe's Team, and Mr. Gordon Tozer. Dr. NM Alajez is a recipient of a scholarship from the Terry Fox Foundation Strategic Training Initiative for Excellence in Radiation Research for the 21st century (EIRR21), at the Canadian Institutes of Health Research (ClHR). Support is also provided from the Campbell Family Institute for Cancer Research and the Ministry of Health and Long-Term Planning.

1. Kleer CG, Cao Q, Varambally S, Shen R, Ota I, Tomlins SA et al. EZH2 is a marker of aggressive breast cancer and promotes neoplastic transformation of breast epithelial cells. Proc Natl Acad Sci USA 2003; 100: 11606-11611.

2. Varambally S, Dhanasekaran SM, Zhou M, Barrette TR, Kumar-Sinha C, Sanda MG et al. The polycomb group protein EZH2 is involved in progression of prostate cancer. Nature 2002; 419: 624-629.

3. Villa R, Pasini D, Gutierrez A, Morey L, Occhionorelli M, Vire E et al. Role of the polycomb repressive complex 2 in acute promyelocytic leukemia. Cancer Cell 2007; 11: 513-525.

4. Levine SS, Weiss A, Erdjument-Bromage H, Shao Z, Tempst P, Kingston RE. The core of the polycomb repressive complex is compositionally and functionally conserved in flies and humans. Mol Cell Biol 2002; 22: 6070-6078.

5. Bracken AP, Kleine-Kohlbrecher D, Dietrich N, Pasini D, Gargiulo G, Beekman C et al. The Polycomb group proteins bind throughout the INK4A-ARF locus and are disassociated in senescent cells. Genes Dev 2007; 21: 525-530.

6. Park IK, Qian D, Kiel M, Becker MW, Pihalja M, Weissman IL et al. Bmi-1 is required for maintenance of adult self-renewing haematopoietic stem cells. Nature 2003; 423: 302-305.

7. Cao R, Zhang $Y$. The functions of $E(Z) / E Z H 2-m e d i a t e d$ methylation of lysine 27 in histone H3. Curr Opin Genet Dev 2004; 14: 155-164.

8. Francis NJ, Saurin AJ, Shao Z, Kingston RE. Reconstitution of a functional core polycomb repressive complex. Mol Cell 2001; 8: 545-556.
9. Calin GA, Croce CM. MicroRNA signatures in human cancers. Nat Rev Cancer 2006; 6: 857-866.

10. Kim VN, Han J, Siomi MC. Biogenesis of small RNAs in animals. Nat Rev Mol Cell Biol 2009; 10: 126-139.

11. Alajez NM, Shi W, Hui AB, Yue S, Ng R, Lo KW et al. Targeted depletion of BMl1 sensitizes tumor cells to P53-mediated apoptosis in response to radiation therapy. Cell Death Differ 2009; 16: 1469-1479.

12. Shi W, Bastianutto C, Li A, Perez-Ordonez B, $\mathrm{Ng} \mathrm{R}$, Chow KY et al. Multiple dysregulated pathways in nasopharyngeal carcinoma revealed by gene expression profiling. Int $\mathrm{J}$ Cancer 2006; 119: 2467-2475

13. Chou TC, Talalay P. Quantitative analysis of dose-effect relationships: the combined effects of multiple drugs or enzyme inhibitors. Adv Enzyme Regul 1984; 22: 27-55.

14. Banath JP, Olive PL. Expression of phosphorylated histone H2AX as a surrogate of cell killing by drugs that create DNA double-strand breaks. Cancer Res 2003; 63: 4347-4350.

15. Bracken AP, Dietrich N, Pasini D, Hansen KH, Helin K. Genome-wide mapping of Polycomb target genes unravels their roles in cell fate transitions. Genes Dev 2006; 20 : 1123-1136.

16. Varambally S, Cao Q, Mani RS, Shankar S, Wang X, Ateeq B et al. Genomic loss of microRNA-101 leads to overexpression of histone methyltransferase EZH2 in cancer. Science 2008; 322: 1695-1699.

17. Bracken AP, Pasini D, Capra M, Prosperini E, Colli E, Helin K. EZH2 is downstream of the pRB-E2F pathway, essential for proliferation and amplified in cancer. EMBO J 2003; 22: 5323-5335.

18. Croonquist PA, Van Ness B. The polycomb group protein enhancer of zeste homolog 2 $(E Z H ~ 2)$ is an oncogene that influences myeloma cell growth and the mutant ras phenotype. Oncogene 2005; 24: 6269-6280.

19. Hui $A B$, Lo KW, Leung SF, Teo P, Fung MK, To KF et al. Detection of recurrent chromosomal gains and losses in primary nasopharyngeal carcinoma by comparative genomic hybridisation. Int J Cancer 1999; 82: 498-503.

20. Sander S, Bullinger L, Klapproth K, Fiedler K, Kestler HA, Barth TF et al. MYC stimulates EZH2 expression by repression of its negative regulator miR-26a. Blood 2008; 112: 4202-4212.

21. Jacob E, Hod-Dvorai R, Schif-Zuck S, Avni O. Unconventional association of the polycomb group proteins with cytokine genes in differentiated Thelper cells. J Biol Chem 2008; 283: 13471-13481.

22. Chatoo W, Abdouh M, David J, Champagne MP, Ferreira J, Rodier F et al. The polycomb group gene Bmi1 regulates antioxidant defenses in neurons by repressing $\mathrm{p} 53$ pro-oxidant activity. J Neurosci 2009; 29: 529-542.

23. Liu J, Cao L, Chen J, Song S, Lee IH, Quijano C et al. Bmi1 regulates mitochondrial function and the DNA damage response pathway. Nature 2009; 459: 387-392.

24. Shi W, Pataki I, MacMillan C, Pintilie M, Payne D, O'Sullivan B et al. Molecular pathology parameters in human nasopharyngeal carcinoma. Cancer 2002; 94: 1997-2006.

25. Banerjee D, Guba A, Guindi M, Haliotis T, Hanna W, Jass J et al. Best practice standards for EGFR testing in colorectal cancer in Canada. SNELL Medical Communication for Bristol-Myers Squibb Canada 2005, Pamphlet, 2005. pp 600-149.

26. Hui AB, Shi W, Boutros PC, Miller N, Pintilie M, Fyles T et al. Robust global micro-RNA profiling with formalin-fixed paraffin-embedded breast cancer tissues. Lab Invest 2009; 89: 597-606.

27. Cheung ST, Huang DP, Hui AB, Lo KW, Ko CW, Tsang YS et al. Nasopharyngeal carcinoma cell line (C666-1) consistently harbouring Epstein-Barr virus. Int J Cancer 1999; 83: $121-126$.

28. Alajez NM, Mocanu JD, Shi W, Chia MC, Breitbach CJ, Hui AB et al. Efficacy of systemically administered mutant vesicular stomatitis virus (VSVDelta51) combined with radiation for nasopharyngeal carcinoma. Clin Cancer Res 2008; 14: 4891-4897.

29. Fenton BM, Paoni SF, Ding I. Pathophysiological effects of vascular endothelial growth factor receptor-2-blocking antibody plus fractionated radiotherapy on murine mammary tumors. Cancer Res 2004; 64: 5712-5719.

30. Gentleman RC, Carey VJ, Bates DM, Bolstad B, Dettling M, Dudoit S et al. Bioconductor: open software development for computational biology and bioinformatics. Genome Biol 2004; 5 : R80.

31. Piontek K, Menezes LF, Garcia-Gonzalez MA, Huso DL, Germino GG. A critical developmental switch defines the kinetics of kidney cyst formation after loss of Pkd1. Nat Med 2007; 13: 1490-1495.

Cell Death and Disease is an open-access journal published by Nature Publishing Group. This work is licensed under the Creative Commons Attribution-Noncommercial-No Derivative Works 3.0 Unported License. To view a copy of this license, visit http://creativecommons.org/licenses/by-nc-nd/3.0/

\section{Supplementary Information accompanies the paper on Cell Death and Disease website (http://www.nature.com/cddis)}

